(c) 2018 - ISSN 1807-2577

\title{
Avaliação de dentes monorradiculares artificiais utilizados para treinamento endodôntico
}

\author{
Evaluation of artificial monoradicular teeth used for endodontic training \\ Juliana da Silva MENDES ${ }^{a}$ (i) , Francisco MONTAGNER $^{b}$ (D), Tiago André Fontoura de MELO ${ }^{b}$ (i), \\ Simone Bonato LUISI ${ }^{\mathrm{b} *}$ (D) \\ aUFRGS - Universidade Federal do Rio Grande do Sul, Porto Alegre, RS, Brasil \\ bUFRGS - Universidade Federal do Rio Grande do Sul, Departamento de Odontologia Conservadora, Área de \\ Endodontia, Porto Alegre, RS, Brasil
}

Como citar: Mendes JS, Montagner F, Melo TAF, Luisi SB. Avaliação de dentes monorradiculares artificiais utilizados para treinamento endodôntico. Rev Odontol UNESP. 2020;49:e20200018. https://doi.org/10.1590/1807-2577.01820

\begin{abstract}
Resumo
Introdução: É desafio do ensino pré-clínico em Endodontia fornecer aos alunos dentes artificiais que reproduzam a morfologia dos dentes humanos. Objetivo: Comparar a morfologia interna e externa de dentes artificiais monorradiculares com dados da literatura sobre dentes humanos. Material e método: Os dentes artificiais foram doados pelos fabricantes: Fábrica de Sorrisos, empresa A, $(n=20)$, e IM do Brasil Ltda., empresa $B,(n=20)$. Foi realizada mensuração do comprimento total do dente e da raiz, da altura, das dimensões vestibulopalatina e mesiodistal da coroa, com paquímetro digital. A descrição morfológica das raízes, coroas e câmaras pulpares foi realizada. A localização topográfica do forame apical principal foi avaliada após exploração dos canais com instrumento endodôntico tipo K \#15 até este atingir visualmente o ápice radicular. Os dados foram dispostos em uma planilha de cálculo e realizou-se a análise estatística [ANOVA e pós-teste de Tukey $(\alpha=5 \%)$ ]. Resultado: A amostra de caninos superiores da empresa $B$ apresentou todas as variáveis dentro dos padrões morfométricos descritos na literatura. Em relação às morfologias interna e externa, a amostra de incisivos laterais superiores da empresa $\mathrm{A}$ foi a única a apresentar todas as variáveis dentro dos padrões descritos na literatura. Em relação à localização topográfica foraminal, os dentes da empresa A, assim como o canino inferior da empresa B, apresentaram tendência à posição distalizada e centralizada, vindo ao encontro da literatura. Conclusão: Nenhum dente artificial estudado apresentou total similaridade morfológica e morfométrica com a literatura analisada, diferindo em pelo menos uma das variáveis, sendo indicada aos fabricantes uma revisão dos seus modelos.
\end{abstract}

Descritores: Dente artificial; educação; endodontia.

\begin{abstract}
Introduction: It is a challenge of pre-clinical teaching in Endodontics to provide students with artificial teeth that reproduce the internal and external morphology of human teeth. Objective: To compare the internal and external morphology of single-rooted artificial teeth with data from the literature on human teeth. Material and method: The artificial teeth were donated from two companies: Fábrica de Sorrisos, company A, $(n=20)$ and IM do Brasil Ltda, company B $(n=20)$. Measurement of total tooth length, root, height, buccal-palatal and mesio-distal crown dimensions with digital caliper was performed. The morphological description of the roots, crown and pulp chamber was performed, the last one only after access. The topographic location of the main apical foramen was evaluated after canal exploration with a $\mathrm{K}$ \#15 endodontic instrument until it visually reached the root apex. The data were arranged in a spreadsheet and statistical analysis was performed (ANOVA, followed by the Tukey post-test $(\alpha=5 \%)$ ). Result: Regarding the morphometric parameters, the upper canine sample from company B presented all variables within the standards described in the literature. Regarding internal and external morphology, the sample of upper lateral incisors from Company A was the only one to present all variables within the standards described in the literature. Regarding the foraminal topographic location, the teeth of Company $\mathrm{A}$, as well as the lower canine of company B, showed a tendency to the distalized and centralized position,
\end{abstract}


in line with the literature. Conclusion: none of the artificial teeth studied showed total morphological and morphometric similarity with the control, differing in at least one of the variables, and a review of their models is indicated to the manufacturers.

Descriptors: Tooth artificial; education; endodontics.

\section{INTRODUÇÃO}

As disciplinas de pré-clínica dos cursos de graduação em Odontologia oportunizam aos graduandos o desenvolvimento de competências e habilidades através de atividades, em manequins, que são preparatórias às atividades clínicas. Na área de Endodontia pré-clínica, são abordados fundamentos teóricos e o desenvolvimento prático da técnica endodôntica, em laboratório, incluindo acesso à câmara pulpar, localização dos canais, preparo químico mecânico e obturação, tanto em dentes anteriores quanto posteriores ${ }^{1-4}$, visando à excelência do método clínico.

Durante muito tempo, as atividades em laboratório foram desenvolvidas em dentes naturais humanos extraídos, coletados sob consentimento informado, os quais, inseridos em manequins, oportunizavam um aprendizado laboratorial semelhante à realidade clínica. Entretanto, por serem dentes provindos de pacientes, eles apresentavam risco à saúde dos alunos devido à possibilidade de infecção cruzada. Hoje, em função das políticas de saúde bucal vigentes, o número de dentes extraídos tem reduzido, devido à diminuição das exodontias. Além disto, nos dentes naturais humanos, observam-se diferentes graus de complexidade operatória devido a variabilidades anatômicas, o que pode ocasionar uma equivocada avaliação do desempenho individual do aluno durante a prática laboratorial ${ }^{1-5}$.

Atualmente, os dentes humanos estão sendo substituídos por dentes artificiais e um dos desafios do ensino pré-clínico é oportunizar aos alunos modelos dentários que possam reproduzir a morfologia externa e o sistema de canais radiculares. Segundo Giongo et al. ${ }^{6}$, em geral, os dentes artificiais são muito semelhantes aos dentes naturais, radiográfica e externamente. Entretanto, o seu uso no ensino pré-clínico de Endodontia deve ser realizado com cautela, devido a limitações observadas, tais como a câmara pulpar reduzida, além de apresentar bolhas e haver discrepâncias na distância entre a região de furca e o soalho da câmara pulpar.

Professores de Endodontia não foram favoráveis ao desenvolvimento do ensino laboratorial em dentes artificiais de duas marcas comerciais brasileiras, após avaliação das suas características morfológicas em tomadas radiográficas digitais ${ }^{7}$.

Essas dificuldades, tanto na morfologia externa quanto interna dos dentes artificiais, suscitam o questionamento acerca da eficiência do aprendizado pré-clínico exclusivamente em modelos artificiais ${ }^{2,8}$. Assim, o presente estudo se propõe a comparar as morfologias interna e externa de dentes artificiais monorradiculares, disponíveis no mercado brasileiro, para treinamento pré-clínico em Endodontia, aos dados da literatura sobre dentes humanos.

\section{MATERIAL E MÉTODO}

O presente estudo foi aprovado pela Comissão de Pesquisa da Faculdade de Odontologia da Universidade Federal do Rio Grande do Sul. Trata-se de um estudo do tipo observacional, descritivo, in vitro.

Quarenta dentes endodônticos artificiais foram obtidos por meio de doação de duas empresas comerciais: Fábrica de Sorrisos (Arujá, São Paulo, Brasil), empresa A, e IM do Brasil Ltda. (São Paulo, São Paulo, Brasil), empresa B. Cada empresa doou cinco dentes de cada grupo dentário: incisivo central superior, incisivo lateral superior, canino superior e canino inferior, resultando em vinte dentes por empresa. 


\section{Cálculo Amostral}

0 tamanho da amostra foi definido com base em estudos prévios da literatura9,10. Para detectar uma diferença de, pelo menos, 1,5 unidade de desvio padrão entre as médias das medidas observadas nos grupos, atingindo um poder estatístico de $90 \%$ com um nível de significância de $5 \%$, foi calculada a necessidade de cinco dentes por grupo.

\section{Obtenção dos Dados Referentes à Morfologia Externa}

Foram mensurados o comprimento total do dente e as dimensões vestibulopalatina (VP) e mesiodistal (MD) da coroa, com ajuda de um paquímetro digital (Mitutoyo, Suzano, São Paulo, Brasil) calibrado em zero milímetro $(0 \mathrm{~mm})$. As aferições foram realizadas nos pontos mais proeminentes das faces. 0 colo dentário foi identificado e este ponto serviu de parâmetro para que fossem aferidos o comprimento da raiz e a altura da coroa, na face vestibular, também com o uso do paquímetro digital. Estas medições foram realizadas três vezes, em dias diferentes, pelo mesmo avaliador.

Também foram realizadas descrições morfológicas da raiz e da coroa dentária em suas faces vestibular e proximal, com auxílio de uma lupa (3,5 vezes de aumento) associada a um fotóforo de led (MMO Optics, São Carlos, São Paulo, Brasil).

\section{Obtenção dos Dados Referentes à Morfologia Interna}

Para avaliação da câmara pulpar, os dentes foram acessados utilizando pontas diamantadas, em alta rotação, número 1012 ou 1014 (KG Sorensen Ind. e Com Ltda., Barueri, São Paulo, Brasil) (Kavo, Joinville, Santa Catarina, Brasil), sob refrigeração.

A forma de contorno da abertura da câmara pulpar foi obtida utilizando a broca Endo Z (Dentsply Ind. e Com Ltda., Petrópolis, Rio de Janeiro, Brasil). A irrigação da câmara pulpar foi realizada com solução de hipoclorito de sódio a 2,5\% (Iodontosul - Industrial Odontológica do Sul Ltda., Porto Alegre, Rio Grande do Sul, Brasil), utilizando uma seringa plástica de $10 \mathrm{~mL}$ (Plastipak Indústria Cirúrgica Ltda., Curitiba, Paraná, Brasil) e agulha Navitip ${ }^{\circledR}$ de $0,30 \mathrm{~mm}$ de diâmetro externo (Ultradent Products, Inc South Jordan, Utah). A exploração dos canais foi realizada com instrumentos endodônticos tipo K \#15 (Dentsply/Maillefer, Ballaigues, Switzerland). Após o acesso e a localização dos canais, foi realizada a descrição da morfologia da câmara pulpar, também com o auxílio da lupa.

A localização do forame principal foi feita através da exploração dos canais com um instrumento endodôntico tipo K \#15 até atingir visualmente o ápice da raiz. Neste momento, foi realizado o registro da posição do instrumento (central, mesial, distal, vestibular ou lingual) em relação ao vértice da raiz, também com o auxílio da lupa.

\section{Obtenção dos Dados Científicos na Literatura Referentes à Morfologia Externa e Interna}

Com relação aos dados morfométricos, foram incluídos neste estudo os achados da literatura de seis autores ${ }^{11-16}$ que realizaram experimentos, nos quais foram mensurados diferentes parâmetros de dentes humanos extraídos. Foi utilizada a média dos dados obtidos, para cada uma das variáveis: comprimento total do dente, comprimento da raiz, altura e dimensões vestibulolingual e mesiodistal da coroa dos dentes humanos permanentes naturais. Em relação aos dados referentes à morfologia externa (formato da raiz, da coroa por vestibular e proximal), estes foram retirados de Della Serra, Ferreira ${ }^{17}$. 
Já em relação à morfologia interna, o formato da câmara pulpar foi classificado de acordo com o relatado por De Deus ${ }^{18}$. Quanto à descrição da localização topográfica foraminal, foram utilizados os achados do estudo de Milano et al. ${ }^{19}$.

\section{Tabulação dos Dados e Análise Estatística}

Os dados obtidos foram distribuídos em uma planilha Excel (Microsoft, 2008) e analisados por meio do programa estatístico GraphPad Prism 8 (GraphPad Software Inc., San Diego, CA, EUA). A normalidade dos dados foi determinada por meio do Teste de Shapiro-Wilk. Para a comparação de cada variável analisada, em um mesmo grupo de dentes de diferentes marcas, utilizou-se o teste de ANOVA de uma via, com pós-teste de Tukey ( $\alpha=5 \%$ ).

A hipótese nula do presente estudo é que não existem diferenças morfológicas e morfométricas entre os dentes artificiais e os dados da literatura referentes aos dentes humanos.

\section{RESULTADO}

\section{Avaliação das Medidas Externas}

Na Figura 1, encontram-se os gráficos referentes aos valores médios das variáveis: comprimento total do dente, comprimento da raiz, altura, dimensões mesiodistal e vestibulolingual da coroa. E, na Tabela 1, encontram-se os valores médios e o desvio padrão das mesmas.

Os incisivos centrais superiores da empresa A não apresentaram diferença estatisticamente significativa com relação ao descrito na literatura, em suas medidas de comprimento total, comprimento da raiz, altura da coroa e dimensões mesiodistal, com exceção da dimensão vestibulolingual da coroa, a qual foi maior que o descrito na literatura. Quanto à empresa $\mathrm{B}$, apenas houve diferença estatística na dimensão mesiodistal da coroa, a qual foi menor que a verificada na literatura.

Os incisivos laterais superiores das empresas A e B não apresentaram diferença estatisticamente significativa com relação à literatura em suas medidas de comprimento total do dente, comprimento da raiz e dimensão mesiodistal da coroa, exceto na altura da coroa e na dimensão vestibulolingual da coroa, as quais foram maiores que as verificadas na literatura.

Os caninos superiores da empresa $B$ não apresentaram diferença estatisticamente significativa com relação ao descrito na literatura, em todas as medidas. Os caninos superiores da empresa A não apresentaram diferença estatisticamente significativa com relação à literatura na altura e dimensão mesiodistal da coroa. Entretanto o comprimento total do dente e o comprimento da raiz foram menores que a literatura e a dimensão vestibulolingual foi maior.

Os caninos inferiores da empresa B não apresentaram diferença estatisticamente significativa com relação à literatura em suas medidas comprimento total, comprimento da raiz, altura da coroa e dimensão vestibulolingual, exceto a dimensão mesiodistal da coroa, que se apresentou menor. Os caninos inferiores da empresa A não apresentaram diferença estatisticamente significativa com relação aos achados da literatura em suas medidas de comprimento da raiz, altura da coroa, dimensões mesiodistal e vestibulolingual, exceto no comprimento total do dente, que foi menor. 


\section{Avaliação Morfológica}

Em relação às morfologias externa e interna, foi utilizada a moda dos resultados para estabelecimento do padrão de cada fabricante, para cada grupo dentário (Tabela 1).

No formato da coroa por vestibular, a marca A apresentou, para todos os grupos dentários, moda idêntica à literatura. No entanto, a marca B apresentou a moda igual à literatura, exceto para o grupo de caninos inferiores, cujo formato se mostrou em forma trapezoidal com tendência retangular, diferentemente da forma pentagonal, apresentada por De Deus ${ }^{18}$.

Em relação ao formato da coroa por proximal, a marca A apresentou apenas igualdade ao descrito na literatura no grupo de incisivos laterais superiores, divergindo em incisivos centrais, caninos inferiores e superiores. Já a marca B apresentou formato igual à literatura nos grupos de incisivos centrais e laterais, apresentando divergência apenas para os caninos inferiores e superiores.

Quanto ao formato da raiz, a empresa A apresentou igualdade à literatura em relação aos dentes incisivo lateral e canino inferior, diferenciando-se nos incisivos centrais e caninos superiores. Entretanto a empresa B apresentou todos seus formatos diferentes da literatura, para todos os grupos dentários.

No que diz respeito à morfologia interna, na avaliação do formato da câmara pulpar, temos os seguintes achados: a empresa $\mathrm{A}$ apresenta formatos iguais à literatura para incisivos centrais $\mathrm{e}$ laterais, porém diverge em caninos superiores e inferiores. A empresa B não apresenta formatos similares de câmara pulpar em nenhum grupo dentário, com relação ao descrito na literatura.

\section{Localização Foraminal}

Na Figura 2, encontra-se a distribuição, em percentual, da localização topográfica do forame apical principal dos diferentes grupos de dentes artificiais, das duas marcas comerciais pesquisadas.

Os dentes da empresa $\mathrm{A}$, assim como o canino inferior da empresa $\mathrm{B}$, apresentaram tendência à posição distalizada e centralizada, vindo ao encontro do estudo de Milano et al. ${ }^{19}$. 

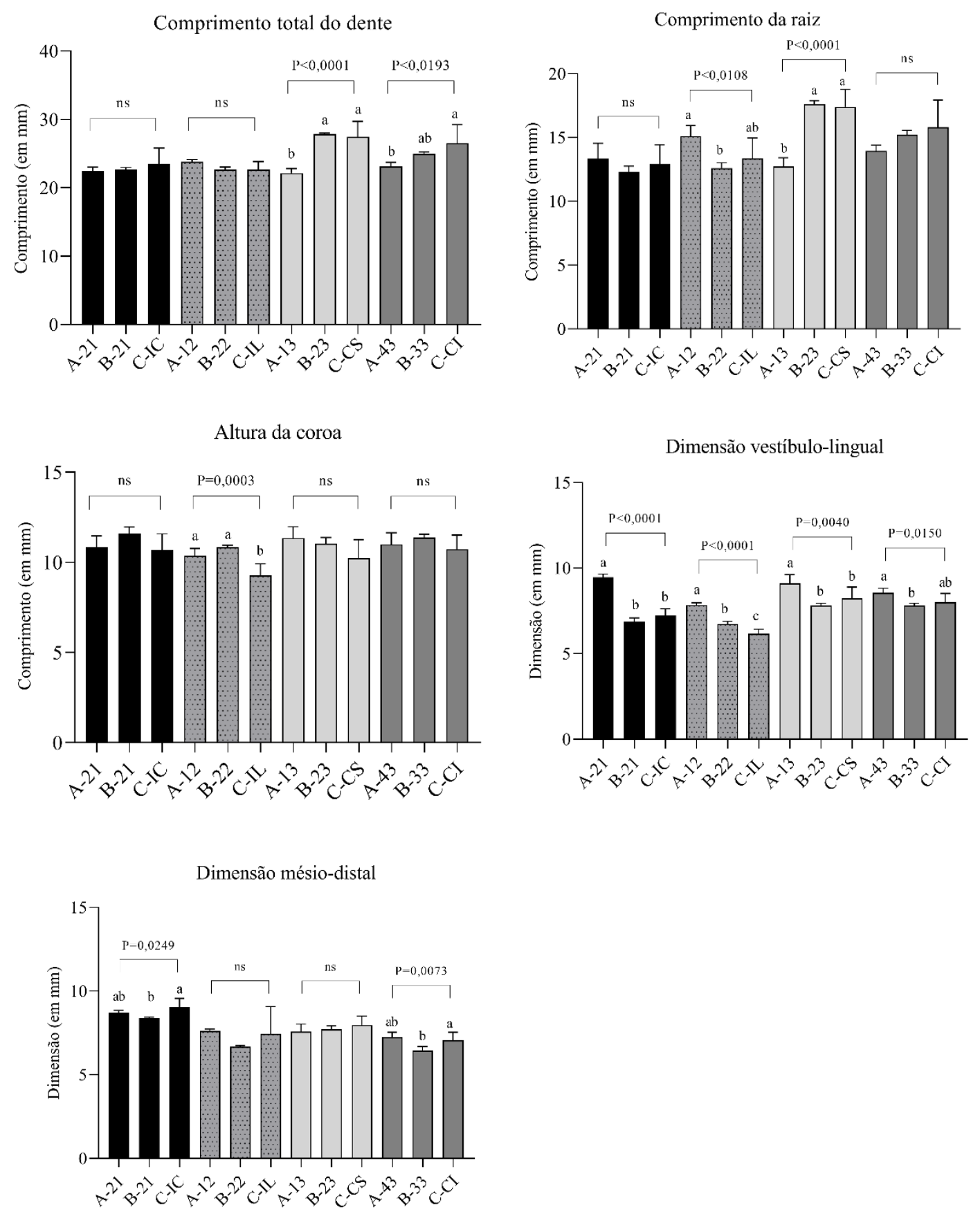

Figura 1. Gráficos referentes aos valores médios das variáveis numéricas analisadas estatisticamente: comprimento total do dente, comprimento da raiz, altura e dimensões mesiodistal e vestibulolingual da coroa. Legenda: A = Fábrica de Sorrisos (Arujá, São Paulo, Brasil); B = IM do Brasil Ltda. (São Paulo, São

Paulo, Brasil); $C$ = Achados da literatura. ns = não houve diferença estatística. Diferentes cores, representam os diferentes grupos dentais (incisivos centrais superiores, dente $21 \mathrm{em}$ ambos os fabricantes; incisivos laterais superiores, dentes 12 e 22; caninos superiores, dentes 13 e 23; caninos inferiores, dentes 43 e 33); $P$ = Probabilidade de significância. Letras minúsculas diferentes em um mesmo grupo dental representam diferença estatisticamente significativa. 


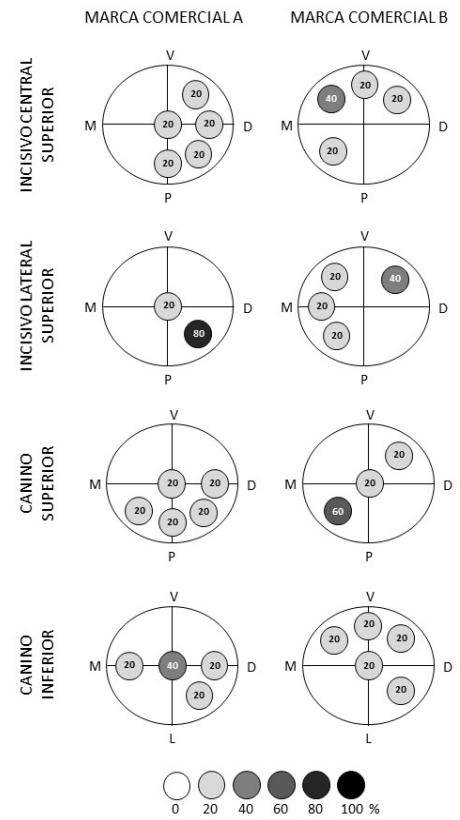

Figura 2. Distribuição, em percentual, da localização topográfica do forame principal dos diferentes grupos de dentes artificiais das duas marcas comerciais pesquisadas. Legenda: $\mathrm{M}=$ Mesial; $\mathrm{V}=$ Vestibular;

$$
\mathrm{D}=\text { Distal; } \mathrm{P}=\text { Palatino; } \mathrm{L}=\text { Lingual. }
$$

Tabela 1. Morfologia externa e interna dos diferentes grupos dentários e média e desvio padrão dos aspectos morfométricos observados nos diferentes grupos experimentais

\begin{tabular}{|c|c|c|c|c|c|c|c|c|c|c|c|c|}
\hline & \multicolumn{3}{|c|}{$\begin{array}{c}\text { Incisivo } \\
\text { Central Superior } \\
\end{array}$} & \multicolumn{3}{|c|}{$\begin{array}{c}\text { Incisivo } \\
\text { Lateral Superior }\end{array}$} & \multicolumn{3}{|c|}{ Canino Superior } & \multicolumn{3}{|c|}{ Canino Inferior } \\
\hline & $\begin{array}{l}\overleftarrow{J} \\
\stackrel{0}{\pi} \\
\sum\end{array}$ & 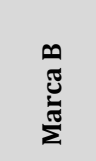 & 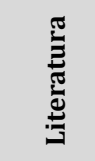 & 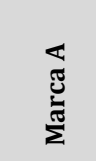 & 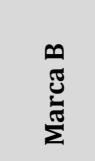 & 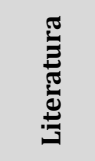 & 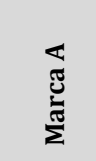 & 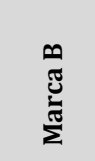 & 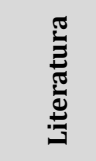 & 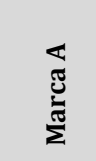 & 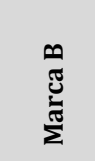 & 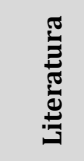 \\
\hline $\begin{array}{c}\text { Formato coroa } \\
\text { vestibular (moda) }\end{array}$ & $1 \mathrm{~V}$ & $1 \mathrm{~V}$ & $1 \mathrm{~V}$ & $1 \mathrm{~V}$ & $1 \mathrm{~V}$ & $1 \mathrm{~V}$ & $4 \mathrm{~V}$ & $4 \mathrm{~V}$ & $4 \mathrm{~V}$ & $4 \mathrm{~V}$ & $3 \mathrm{~V}$ & $4 \mathrm{~V}$ \\
\hline $\begin{array}{c}\text { Formato coroa } \\
\text { proximal (moda) }\end{array}$ & $2 \mathrm{P}$ & $1 \mathrm{P}$ & $1 \mathrm{P}$ & $1 \mathrm{P}$ & $1 \mathrm{P}$ & $1 \mathrm{P}$ & $2 \mathrm{P}$ & $2 \mathrm{P}$ & $3 \mathrm{P}$ & $2 \mathrm{P}$ & $2 \mathrm{P}$ & $3 P$ \\
\hline $\begin{array}{l}\text { Formato raiz } \\
\text { (moda) }\end{array}$ & $3 \mathrm{R}$ & $2 \mathrm{R}$ & $1 \mathrm{R}$ & $3 \mathrm{R}$ & $2 \mathrm{R}$ & $3 \mathrm{R}$ & $3 R$ & $2 \mathrm{R}$ & $1 \mathrm{R}$ & $3 \mathrm{R}$ & $2 \mathrm{R}$ & $3 \mathrm{R}$ \\
\hline $\begin{array}{l}\text { Formato câmara } \\
\text { pulpar (moda) }\end{array}$ & $4 \mathrm{C}$ & $2 \mathrm{C}$ & $4 \mathrm{C}$ & $4 \mathrm{C}$ & $2 \mathrm{C}$ & $4 \mathrm{C}$ & $3 \mathrm{C}$ & $3 \mathrm{C}$ & $5 \mathrm{C}$ & $1 \mathrm{C}$ & $2 \mathrm{C}$ & $6 \mathrm{C}$ \\
\hline $\begin{array}{l}\text { Comprimento total } \\
\text { (mm) }\end{array}$ & $\begin{array}{c}22,5 \\
( \pm 0,6)\end{array}$ & $\begin{array}{c}22,7 \\
( \pm 0,3)\end{array}$ & $\begin{array}{c}23,5 \\
( \pm 2,1)\end{array}$ & $\begin{array}{c}23,7 \\
( \pm 0,3)\end{array}$ & $\begin{array}{c}22,6 \\
( \pm 0,3)\end{array}$ & $\begin{array}{c}22,6 \\
( \pm 1,1)\end{array}$ & $\begin{array}{c}22,1 \\
( \pm 0,7)\end{array}$ & $\begin{array}{c}27,8 \\
( \pm 0,2)\end{array}$ & $\begin{array}{c}27,4 \\
( \pm 2,1)\end{array}$ & $\begin{array}{c}23,1 \\
( \pm 0,6)\end{array}$ & $\begin{array}{c}24,9 \\
( \pm 0,3)\end{array}$ & $\begin{array}{c}26,5 \\
( \pm 2,5)\end{array}$ \\
\hline $\begin{array}{l}\text { Comprimento da } \\
\text { raiz }(\mathrm{mm})\end{array}$ & $\begin{array}{c}13,3 \\
( \pm 1,2)\end{array}$ & $\begin{array}{c}12,2 \\
( \pm 0,4)\end{array}$ & $\begin{array}{c}12,9 \\
( \pm 1,5)\end{array}$ & $\begin{array}{c}15,0 \\
( \pm 0,8)\end{array}$ & $\begin{array}{c}12,5 \\
( \pm 0,4)\end{array}$ & $\begin{array}{c}13,3 \\
( \pm 1,5)\end{array}$ & $\begin{array}{c}12,7 \\
( \pm 0,6)\end{array}$ & $\begin{array}{l}17,6 \\
(0,2)\end{array}$ & $\begin{array}{c}17,4 \\
( \pm 1,3)\end{array}$ & $\begin{array}{l}13,9 \\
( \pm 0,4)\end{array}$ & $\begin{array}{c}15,2 \\
( \pm 0,3)\end{array}$ & $\begin{array}{c}15,2 \\
( \pm 0,3)\end{array}$ \\
\hline $\begin{array}{l}\text { Altura da coroa } \\
\text { (mm) }\end{array}$ & $\begin{array}{c}10,8 \\
( \pm 0,6)\end{array}$ & $\begin{array}{l}11,6 \\
( \pm 0,3)\end{array}$ & $\begin{array}{c}10,6 \\
( \pm 0,8)\end{array}$ & $\begin{array}{c}10,3 \\
( \pm 0,4)\end{array}$ & $\begin{array}{c}10,8 \\
( \pm 0,1)\end{array}$ & $\begin{array}{c}9,2 \\
( \pm 0,6)\end{array}$ & $\begin{array}{l}11,3 \\
(0,6)\end{array}$ & $\begin{array}{l}11,0 \\
( \pm 0,3)\end{array}$ & $\begin{array}{c}10,2 \\
( \pm 1,0)\end{array}$ & $\begin{array}{c}10,9 \\
( \pm 0,6)\end{array}$ & $\begin{array}{l}11,3 \\
( \pm 0,2)\end{array}$ & $\begin{array}{c}10,7 \\
( \pm 0,7)\end{array}$ \\
\hline Largura VL (mm) & $\begin{array}{c}9,4 \\
( \pm 0,1)\end{array}$ & $\begin{array}{c}6,8 \\
( \pm 0,2)\end{array}$ & $\begin{array}{c}7,2 \\
( \pm 0,2)\end{array}$ & $\begin{array}{c}7,8 \\
( \pm 0,1)\end{array}$ & $\begin{array}{c}6,7 \\
( \pm 0,1)\end{array}$ & $\begin{array}{c}6,1 \\
( \pm 0,2)\end{array}$ & $\begin{array}{c}9,1 \\
( \pm 0,5)\end{array}$ & $\begin{array}{c}7,8 \\
( \pm 0,1)\end{array}$ & $\begin{array}{c}8,2 \\
( \pm 0,6)\end{array}$ & $\begin{array}{c}8,5 \\
( \pm 0,2)\end{array}$ & $\begin{array}{c}7,8 \\
( \pm 0,1)\end{array}$ & $\begin{array}{c}8,0 \\
( \pm 0,5)\end{array}$ \\
\hline Largura MD (mm) & $\begin{array}{c}8,7 \\
( \pm 0,1)\end{array}$ & $\begin{array}{c}8,3 \\
( \pm 0,0)\end{array}$ & $\begin{array}{c}9,0 \\
( \pm 0,5)\end{array}$ & $\begin{array}{c}7,6 \\
( \pm 1,1)\end{array}$ & $\begin{array}{c}6,6 \\
( \pm 0,0)\end{array}$ & $\begin{array}{c}7,4 \\
( \pm 1,6)\end{array}$ & $\begin{array}{c}7,5 \\
( \pm 0,4)\end{array}$ & $\begin{array}{c}7,7 \\
( \pm 0,2)\end{array}$ & $\begin{array}{c}7,9 \\
( \pm 0,5)\end{array}$ & $\begin{array}{c}7,2 \\
( \pm 0,2)\end{array}$ & $\begin{array}{c}6,6 \\
( \pm 0,2)\end{array}$ & $\begin{array}{c}7,0 \\
( \pm 0,4)\end{array}$ \\
\hline
\end{tabular}

Legenda: 1V: Trapezoidal, com base incisal alargada no sentido MD; 3V: Trapezoidal, com tendência retangular; 4V: Pentagonal; 1P: Cuneiforme, forte achatamento no sentido VL; 2P: Cuneiforme, suave achatamento no sentido VL; 3P: Triangular; 1R: Cônica; 2R: Triangular prismática; 3R: Cônica, achatada no sentido MD; 1C: Ausente; 2C: Presente, extensão do canal radicular com formato circular; 3C: Presente, achatada VL com paredes proximais paralelas; 4C: Presente, achatada VL com formato triangular, alargada MD na incisal; 5C: Presente, maior diâmetro VL, afilada em sentido oclusal; 6C: Presente, maior diâmetro VL, achatada MD, afilada em sentido oclusal. 


\section{DISCUSSÃO}

A hipótese nula do presente estudo de que não existiriam diferenças morfológicas e morfométricas entre os dentes artificiais e os dados da literatura de dentes humanos foi rejeitada. Nenhum dos grupos dentários avaliados apresentou similaridade em ambos os padrões morfológicos e morfométricos com os dados da literatura dos dentes humanos.

Em relação aos aspectos morfométricos, os dentes de ambos os fabricantes apresentam pelo menos uma dimensão diferente do descrito na literatura, com exceção do canino superior da empresa B (Figura 1). Cabe salientar que os dentes naturais também sofrem variações nas suas dimensões, que podem ser influenciadas por diversos aspectos, como sexo, idade, raça e tipo cefálico do indivíduo ${ }^{17}$, sendo pequenas variações aceitáveis com relação ao tamanho dos dentes artificiais. Entretanto, estas pequenas variações não podem descaracterizar a morfologia externa dentária e, sim, respeitar certa proporcionalidade. As duas marcas comerciais poderiam adequar as dimensões dentárias arbitrárias à literatura de tal forma a garantir a proporcionalidade e a morfologia externa adequadas.

Segundo Della Serra, Ferreira ${ }^{17}$, a cavidade pulpar repete, em proporções menores, a forma exterior do dente. Com relação aos dentes artificiais estudados, a forma da coroa por vestibular foi adequada à literatura em todos os dentes, com exceção do canino inferior da empresa B. Já a forma da coroa por proximal apresentou maior variação de resultados. As morfologias da coroa por vestibular e por proximal deveriam estar diretamente relacionadas com o formato da câmara pulpar. Entretanto, não foi observada correlação entre as anatomias externa e interna dos dentes artificiais, com exceção do incisivo lateral superior da empresa A.

0 formato adequado da câmara pulpar é de fundamental importância para o exercício préclínico da abertura coronária. Um aspecto relevante que norteia os estudantes durante a abertura da câmara pulpar é a sensação conhecida como "cair no vazio". A falta desta sensação e a ausência da câmara pulpar podem levar ao sentimento de insegurança, pois o aluno perde a referência dos limites entre teto e soalho da câmara pulpar ${ }^{1,20}$.

A câmara pulpar dos dentes da empresa B diferiram dos achados da literatura em todos os grupos dentários. Nos incisivos e no canino inferior, a câmara pulpar caracterizava-se por ser uma extensão do canal radicular com formato circular. Por sua vez, no canino superior, apresentavase com formato achatado vestibulolingual e paredes proximais paralelas, diferindo da literatura, segundo a qual a câmara pulpar apresenta-se com maior diâmetro vestibulolingual e afilado no sentido oclusal.

Nos incisivos centrais e laterais da empresa A, a câmara pulpar apresentou-se igual à literatura. Por outro lado, o canino inferior não apresentou câmara pulpar e o canino superior apresentou câmara pulpar achatada no sentido vestibulolingual e paredes proximais paralelas em ambos os caninos, diferindo da literatura.

De acordo com Milano et al. ${ }^{19}$, há uma tendência do forame apical principal apresentar-se em posição distalizada, podendo ainda dirigir-se para as posições distovestibular e distolingual, concordando com os resultados apresentados pelas amostras da empresa A. Por outro lado, à exceção do canino superior, que apresentou o forame na posição mesiopalatina em $60 \%$ dos casos, os demais dentes da empresa B apresentaram forames entre 60 e $80 \%$ vestibularizados, e os formatos das raízes em todos os grupos dentários apresentaram-se diferentes do descrito na literatura. Já na empresa A o incisivo lateral superior e o canino inferior apresentaram os formatos das raízes similares à literatura, sendo divergentes nos dentes canino superior e incisivo central.

Assim, sugere-se que os dentes artificiais avaliados possam sofrer adequações em sua morfologia externa e interna com o intuito de apresentar maior semelhança com o dente natural. Dessa forma, seria oferecida aos alunos de pré-clínica a oportunidade de um ensino mais próximo o possível da realidade clínica, otimizando o aprendizado endodôntico. 


\section{CONCLUSÃO}

Nenhum dente artificial estudado apresentou total similaridade morfológica e morfométrica com o descrito na literatura, demonstrando diferença em pelo menos uma das variáveis. Dentro dos grupos dentários estudados, o incisivo lateral superior da empresa A foi o que mais se aproximou do modelo humano, pois apresentou correlação entre as morfologias interna e externa.

\section{AGRADECIMENTOS}

Agradecemos às empresas Fábrica de Sorrisos e IM do Brasil Ltda pela doação dos dentes utilizados neste estudo.

\section{REFERÊNCIAS}

1. Al-Sudani DI, Basudan SO. Students' perceptions of pre-clinical endodontic training with artificial teeth compared to extracted human teeth. Eur J Dent Educ. 2017 Nov;21(4):e72-5. http://dx.doi.org/10.1111/eje.12223. PMid:27495270.

2. Bitter $\mathrm{K}$, Gruner $\mathrm{D}$, Wolf $\mathrm{O}$, Schwendicke $\mathrm{F}$. Artificial versus natural teeth for preclinical endodontiv training: a randomized controlled trial. J Endod. 2016 Aug;42(8):1212-7. http://dx.doi.org/10.1016/j.joen.2016.05.020. PMid:27469437.

3. Tchorz JP, Brandl M, Ganter PA, Karygianni L, Polydorou O, Vach K, et al. Pre-clinical endodontic training with artificial instead of extracted human teeth: does the type of exercise have na influence on clinical endodontic outcomes? Int Endod J. 2015 Sept;48(9):888-93. http://dx.doi.org/10.1111/iej.12385. PMid:25266846.

4. Robberecht L, Chai F, Dehurtevent M, Marchandise P, Bécavin T, Hornez J-C, et al. A novel anatomical ceramic root canal simulator for endodontic training. Eur J Dent Educ. 2017 Nov;21(4):e1-6. http://dx.doi.org/10.1111/eje.12207. PMid:27146633.

5. Nassri MRG, Carlik J, Silva CRN, Okagawa RE, Lin S. Critical analysis of artificial teeth for endodontic teaching. J Appl Oral Sci. 2008 Jan-Feb;16(1):43-9. http://dx.doi.org/10.1590/S167877572008000100009. PMid:19089288.

6. Giongo M, Gaona P, Victorino FR. Anatomical analysis of the pulp chamber of artificial teeth. RSBO (Online). 2016 Jul-Set;13(3):194-8. http://dx.doi.org/10.21726/rsbo.v13i3.314.

7. Weschenfelder VM, Bainy PT, Vizzotto MB, Luisi SB, Montagner F, Melo TAF. Radiopacidade de dentes artificiais para treinamento pré-clínico de endodontia. Rev Odontol UNESP. 2019;48:e20190053. http://dx.doi.org/10.1590/1807-2577.05319.

8. Narayanaraopeta U, Alshwaimi E. Preclinical endodontic teaching: a survey of Saudi dental schools. Saudi Med J. 2015 Jan;36(1):94-100. http://dx.doi.org/10.15537/smj.2015.1.9336. PMid:25630011.

9. Medeiros JMF, Ferreira GS, Habitante SM, Von Dollinger CFA, Forghieri AA, Haddad Filho MS. Análise da dureza vickers da superfície de dentes artificiais em resina poliéster. Rev Odontol Univ Cid. 2014 Jan-Abr;26(1):23-37.

10. Medeiros JMF, Naregi ES, Almeida ETDC, Von Dollinger CFA, Rosa LCL, Haddad Filho S, et al. Medidas de dureza Vickers na superfície de blocos de resina fenólica com canais endodônticos simulados. Publ UEPG Ciênc Biol Saúde. 2014;20(1):53-71. http://dx.doi.org/10.5212/Publ.Biologicas.v.20i1.0006.

11. Figún MD. Anatomia odontológica funcional e aplicada. São Paulo: Panamericana; 1994.

12. Black GV. Descriptive anatomy of the human teeth. 4th ed. Philadelphia: The S. S. White Dental Manufac.; 1897. 
13. Bouland A, Lebourg L. Menuel de dessin dentaire. 3rd ed. Paris: Masson et Cie.; 1932.

14. Diamond M. Dental anatomy. New York: The MacMillian; 1952.

15. Marseillier E. Les dents humaines. Paris: Gauthier Villars; 1937.

16. Sicher H, Tandler J. Anatomy for dentists. São Paulo: Atheneu; 1981.

17. Della Serra O, Ferreira FV. Anatomia dental. 3. ed. São Paulo: Artes Médicas; 1981.

18. De Deus QD. Endodontia. 5. ed. Rio de Janeiro: MEDSI; 1992.

19. Milano NF, Werner SM, Kapczinski M. Location of the principal foramen. True location versus methods using conductometry. RGO. 1983 Jul-Set;31(3):220-4. PMid:6592697.

20. Luz DS, Ourique FS, Scarparo RK, Vier-Pelisser FV, Morgental RD, Waltrick SB, et al. Preparation time and perceptions of Brazilian specialists and dental students regarding simulated root canals for endodontic teaching: a preliminar study. J Dent Educ. 2015 Jan;79(1):56-63.

http://dx.doi.org/10.1002/j.0022-0337.2015.79.1.tb05857.x. PMid:25576553.

\section{CONFLITOS DE INTERESSE}

Os autores declaram não haver conflitos de interesse.

\section{*AUTOR PARA CORRESPONDÊNCIA}

Simone Bonato Luisi, UFRGS - Universidade Federal do Rio Grande do Sul, Departamento de Odontologia Conservadora, Área de Endodontia, Rua Ramiro Barcelos, 2492, Bairro Santana, 90035-003 Porto Alegre - RS, Brasil, e-mail: simone.luisi@ufrgs.br

Recebido: Março 24, 2020

Aprovado: Maio 28, 2020 